\title{
OFDM Aided Space-Time Shift Keying for Dispersive Downlink Channels
}

\author{
Marco Driusso, Fulvio Babich \\ Dipartimento di Ingegneria Industriale e dell'Informazione \\ Università di Trieste, Via A. Valerio 10, 34127 Trieste, Italy \\ Email:s165518@stud.units.it,babich@units.it
}

\author{
Mohammad Ismat Kadir, Lajos Hanzo \\ School of Electronics and Computer Science \\ University of Southampton, SO17 1BJ, UK \\ Email: \{miklg09,1h\}@ecs.soton.ac.uk
}

\begin{abstract}
The performance of a Space-Time Shift Keying (STSK) scheme was shown to degrade in frequency-selective fading channels. Hence, we propose Orthogonal Frequency Division Multiplexing (OFDM) combined with STSK for frequencyselective broadband channels. Furthermore, we consider both an uncoded and a near-capacity coded scenario. Our results show that a STSK system combined with OFDM is capable of overcoming the impairments of dispersive channels, hence approaching the same performance as in a flat-fading channel.
\end{abstract}

\section{INTRODUCTION}

Multiple-Input Multiple-Output (MIMO) systems have attracted significant interest, since they support reliable highthroughput communications within the same bandwidth as a Single-Input Single-Output system. MIMO techniques allow us either to increase the systems throughput [1] or to improve the systems diversity gain [2], [3].

The concept of Linear Dispersion Codes (LDC) [4] was introduced as a general framework for designing Space-Time Codes that are capable of exploiting both the multiplexing and diversity gain offered by a MIMO system, at the cost of a potentially high encoding complexity. Encouraged by the concept of low-complexity systems such as Spatial Modulation (SM) [5] and Space-Shift Keying (SSK) [6], Sugiura et al. proposed the Space-Time Shift Keying framework [7] as a system capable of providing both multiplexing and transmit diversity gains in a narrowband scenario, whilst exhibiting a lower encoding and decoding complexity than LDCs.

However, in a frequency-selective environment the STSK system suffers from the ISI imposed by the channel. In [8] the authors proposed Space-Time-Frequency Shift Keying (STFSK) as a technique capable of overcoming the effects of dispersive channels, while striking the required diversitymultiplexing trade-offs. STFSK is a technique capable of exploiting time, space and frequency diversity, hence attaining a good performance even in a broadband channel. The frequency dimension is exploited using Frequency Shift Keying (FSK) modulation at the transmitter and a square-law detector at the receiver. Nevertheless, this realization of the STFSK scheme suffers from the typical drawbacks of non-coherent FSK demodulation. These impediments of STFSK may be

The financial support of the EPSRC-UK under the auspices of the IndiaUK Advanced Technology Centre, as well as of the China-UK Science Bridge and that of the EU's Concerto Project, is gratefully acknowledged. eliminated by reduced-rate OFDM-style parallel transmissions over numerous non-dispersive sub-carriers.

This paper proposes a novel modality of realizing spacetime coding designed for broadband channels using Orthogonal Frequency Division Multiplexing (OFDM) combined with the STSK scheme. More particularly, the OFDM modem transforms the Time Domain (TD) spreading operation of the STSK scheme to Frequency Domain (FD) spreading, since every sub-carrier of the OFDM modulation carries a column of each STSK codeword. This allows us to overcome the typical broadband channel's impairments. The performance of the proposed scheme is evaluated using Monte Carlo simulations. Our results demonstrate that the new OFDMaided STSK scheme outperforms the original STSK scheme in broadband channels, and may approach the performance of the narrowband STSK scheme, both in the channel-coded and in the uncoded scenario.

The outline of this paper is as follows. Section II describes the system architecture, the channel model adopted, the en/decoding procedure and the design of the dispersion matrices. In Section III our performance results are discussed. Finally, in Section IV we provide our conclusions, followed by ideas for future research.

\section{SYSTEM OVERVIEW}

The key idea of the proposed OFDM-aided STSK system is to use the OFDM technique in order to create a number of parallel FD sub-channels, where the sub-channel bitrate is sufficiently low for the sub-channel symbol to avoid dispersion.

The resultant system employs an OFDM modulator having $N_{c}$ sub-carriers and a sampling interval of $\mathcal{T}$ at the output.

\section{A. Transmission}

The model of [7] has been adopted for the STSK system while the model of [9] has been used for realizing a MIMOOFDM scheme. Figure 1 outlines the system's architecture. The OFDM-aided STSK encoding process is as follows:

1) the encoder is fed with a block of $B=\log _{2}(\mathcal{L} Q)$ bits, which are spread over $T$ time slots of an STSK symbol; the $\log _{2}(Q)$ bits select one of the $Q$ space-time matrices taken from the set $\left\{\boldsymbol{A}_{q}\right\}_{q=1}^{Q}$, where we have $\boldsymbol{A}_{q} \in \mathbb{C}^{M \times T} \forall q ; \log _{2}(\mathcal{L})$ bits correspond to a specific symbol in a $\mathcal{L}$-PSK/QAM constellation $\mathcal{C}_{\mathcal{L}}$; 


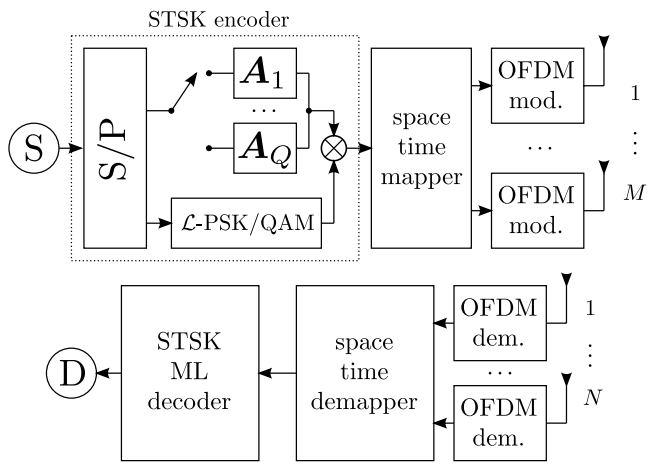

Figure 1. Architecture of the proposed OFDM-aided STSK system.

2) the STSK codeword is then $\boldsymbol{X}=\boldsymbol{A}_{q} s_{l}$, where $s_{l} \in \mathcal{C}_{\mathcal{L}}$ and $\boldsymbol{A}_{q} \in\left\{\boldsymbol{A}_{q}\right\}_{q=1}^{Q} ; \mathcal{C}_{\mathcal{L}}$ has to be a unity-energy constellation, where the space-time dispersion matrices have to satisfy the power constraint of $\operatorname{tr}\left(\boldsymbol{A}_{q}^{\mathrm{H}} \boldsymbol{A}_{q}\right)=T \forall q$ in order to have unity energy for each sub-carrier.

We will refer to this system as an OFDM-aided $\operatorname{STSK}(M, N, T, Q, \mathcal{L})$ scheme, where $M$ is the number of transmit antennas, $N$ is the number of receive antennas, and the space-time matrices are used for spreading an $\mathcal{L}$-ary symbol to a $T$-time slot STSK symbol.

The transmission regime is then organized as follows. The space-time mapper collects $J$ space-time codewords, where $J=N_{c} / T\left(N_{c}\right.$ has to be a multiple of $\left.T\right)$. After $J$ STSK symbol intervals the space-time mapper collected the frame $\mathcal{F}_{T}$, defined as the matrix:

$$
\mathcal{F}_{T}=[\boldsymbol{X}(1) \boldsymbol{X}(2) \ldots \boldsymbol{X}(J)] \in \mathbb{C}^{M \times N_{c}},
$$

where $\boldsymbol{X}(j)$ is the $j^{\text {th }}$ space-time codeword in the frame. The frame $\mathcal{F}_{T}$ can also be written as:

$$
\mathcal{F}_{T}=\left[\tilde{\mathbf{S}}[0] \tilde{\mathbf{S}}[1] \ldots \tilde{\mathbf{S}}\left[N_{c}-1\right]\right] \in \mathbb{C}^{M \times N_{c}}
$$

where $\tilde{\mathbf{S}}\left[n_{c}\right] \in \mathbb{C}^{M \times 1}$ for every $n_{c}=0, \ldots,\left(N_{c}-1\right)$ represents the columns of $\mathcal{F}_{T}$. Hence the vector $\tilde{\mathbf{S}}\left[n_{c}\right]$ is:

$$
\tilde{\mathbf{S}}\left[n_{c}\right]=\left(\boldsymbol{X}\left(\left\lceil n_{c} / T\right\rceil\right)\right)_{\left\langle n_{c}\right\rangle_{T}+1} n_{c}=0, \ldots,\left(N_{c}-1\right),
$$

implying that the $\left(\left\langle n_{c}\right\rangle_{T}+1\right)^{\text {th }}$ column of the $\left\lceil n_{c} / T\right\rceil^{\text {th }}$ space-time codeword in the frame $\mathcal{F}_{T}$ is transmitted via the $n_{c}{ }^{\text {th }}$ sub-carrier of the OFDM system, where the $(\cdot)_{t}$ operator selects the $t^{\text {th }}$ column of a matrix, $\langle\cdot\rangle_{N}$ represents the modulo- $N$ operator and $\lceil\cdot]$ is the ceiling operator. The $m^{\text {th }}$ component of the vector $\tilde{\mathbf{S}}\left[n_{c}\right]$ is denoted with $\tilde{S}_{m}\left[n_{c}\right]$, hence $\tilde{\mathbf{S}}\left[n_{c}\right]=\left[\tilde{S}_{1}\left[n_{c}\right] \tilde{S}_{2}\left[n_{c}\right] \ldots \tilde{S}_{M}\left[n_{c}\right]\right]^{T}$ (where $(\cdot)^{T}$ is the transpose operator). The $N_{c}$ symbols $\left\{\tilde{S}_{m}\left[n_{c}\right]\right\}_{n_{c}=0}^{N_{c}-1}$ are a length- $N_{c}$ sequence that is denoted with $\left\{\tilde{S}_{m}\right\}$. As explained in Figure 2, the sequence $\left\{\tilde{S}_{m}\right\}$ is OFDM-modulated using a classic IFFT-based OFDM modulator, resulting in the sequence:

$$
\tilde{s}_{m}\left[n_{c}\right]=\sqrt{N_{c}} \cdot \operatorname{IDFT}\left\{\tilde{S}_{m}\left[n_{c}\right]\right\} \quad n_{c}=0, \ldots,\left(N_{c}-1\right),
$$

for each transmit antenna $m=1, \ldots, M$. The sequences $\left\{\tilde{s}_{m}\left[n_{c}\right]\right\}_{n_{c}=0}^{N_{c}-1} \quad \forall m$ can be grouped in the sequence of vectors $\left\{\tilde{\mathbf{s}}\left[n_{c}\right]\right\}_{n_{c}=0}^{N_{c}-1} \in \mathbb{C}^{M \times 1}$, where $\tilde{\mathbf{s}}\left[n_{c}\right]=$

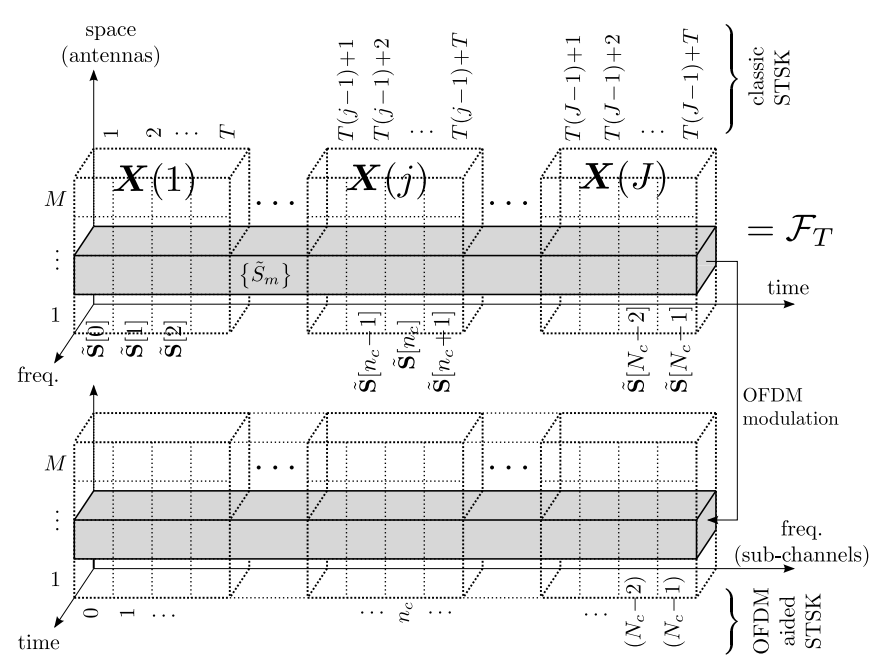

Figure 2. In the classic STSK framework the $t^{\text {th }}$ column of the $j^{\text {th }}$ spacetime codeword is transmitted during the $(T \cdot(j-1)+t)^{\text {th }}$ time interval. In our OFDM-aided STSK system $J$ space-time codeword are transmitted at the same time in one OFDM frame, by spreading the codewords in the frequency domain. More particularly, OFDM modulation is performed across the rows of the frame $\mathcal{F}_{T}$, which are the length- $N_{c}$ sequences $\left\{\tilde{S}_{m}\right\}$. Thus the column $\tilde{\mathbf{S}}\left[n_{c}\right]$ of the transmission frame $\mathcal{F}_{T}$ is transmitted through the $n_{c}^{\text {th }}=(T \cdot(j-1)+t)^{\text {th }}$ sub-channel.

$\left[\tilde{s}_{1}\left[n_{c}\right] \tilde{s}_{2}\left[n_{c}\right] \ldots \tilde{s}_{M}\left[n_{c}\right]\right]^{T} n_{c}=0, \ldots,\left(N_{c}-1\right)$, which represents the $N_{c}$ TD samples of each of the $M$ OFDM symbols transmitted by the $M$ transmit antennas.

The usual Cyclic Prefix (CP) is attached to each TD OFDM symbol of $\left\{\tilde{\mathbf{s}}\left[n_{c}\right]\right\}_{n_{c}=0}^{N_{c}-1}$, leading to the signal vector $\left.\left\{\tilde{\mathbf{s}}_{\mathrm{cp}}\left[n_{c}\right]\right\}\right\}_{n_{c}=0}^{N_{c}-1}$, which is transmitted through the frequencyselective time-variant MIMO channel. Each component of the signal vector $\left\{\tilde{\mathbf{s}}_{\mathrm{cp}}\left[n_{c}\right]\right\}_{n_{c}=0}^{N_{c}-1}$ is given by Eq. (5) for every transmit antenna $m=1, \ldots, M$. The parameter $L_{\mathrm{cp}}=L_{\mathrm{ch}}-1$ in (5) is the length of the CP and $L_{\mathrm{ch}}$ is the length of the FIR filter that models the frequency selective channel.

\section{B. System's Throughput}

In the presence of the $\mathrm{CP}$ the proposed scheme transmits $J$ space-time codewords every $\mathcal{T}\left(N_{c}+L_{\mathrm{cp}}\right)$ seconds. Every OFDM symbol carries $J=N_{c} / T$ STSK codewords and every space-time codeword carries $\log _{2}(\mathcal{L} Q)$ bits. Hence the system's throughput is:

$$
R_{\mathrm{cp}}=\frac{\log _{2}(\mathcal{L} Q)}{T\left(1+L_{\mathrm{cp}} / N_{c}\right)} \quad\left[\frac{\text { bit info }}{\text { symbol }}\right] .
$$

For a sufficiently high number of sub-carriers $N_{c}$ the effect of the $\mathrm{CP}$ on the throughput may be neglected, being:

$$
R=\lim _{N_{c} \rightarrow \infty} R_{\mathrm{cp}}=\frac{\log _{2}(\mathcal{L} Q)}{T} \quad\left[\frac{\text { bit info }}{\text { symbol }}\right] .
$$

\section{The OFDM-STSK Receiver}

Let us consider a low mobility frequency-selective environment in which the channel's envelope remains constant during an OFDM symbol. Then the discrete-time CIR of such frequency-selective MIMO channel may be modelled as:

$$
\mathbf{H}\left[n_{c}\right]=\sum_{l=0}^{L_{\mathrm{ch}}-1} \mathbf{H}[l] \delta\left[n_{c}-l\right] \in \mathbb{C}^{N \times M},
$$




$$
\begin{gathered}
\left\{\tilde{s}_{m, \mathrm{cp}}\left[n_{c}\right]\right\}_{n_{c}=0}^{N_{c}+L_{\mathrm{cp}}-1}=\left\{\tilde{s}_{m}\left[N_{c}-L_{\mathrm{cp}}\right] \ldots \tilde{s}_{m}\left[N_{c}-1\right] \tilde{s}_{m}[0] \tilde{s}_{m}[1] \ldots \tilde{s}_{m}\left[N_{c}-1\right]\right\} \quad m=1, \ldots, M \\
L_{e}\left(x_{b}\right)=\log \left\{\frac{\sum_{\boldsymbol{K}_{h} \in \mathbb{K}_{0}^{b}} \exp \left[-\gamma\left\|\overline{\boldsymbol{Y}}(j)-\overline{\boldsymbol{H}}_{\mathrm{eq}}^{j} \chi \boldsymbol{K}_{h}\right\|^{2}-\sum_{b^{\prime}=1, b^{\prime} \neq b}^{B} x_{b^{\prime}}^{h} L_{a}\left(x_{b^{\prime}}\right)\right]}{\sum_{\boldsymbol{K}_{h} \in \mathbb{K}_{1}^{b}} \exp \left[-\gamma\left\|\overline{\boldsymbol{Y}}(j)-\overline{\boldsymbol{H}}_{\mathrm{eq}}^{j} \chi \boldsymbol{K}_{h}\right\|^{2}-\sum_{b^{\prime}=1, b^{\prime} \neq b}^{B} x_{b^{\prime}}^{h} L_{a}\left(x_{b^{\prime}}\right)\right]}\right\} \quad b=1, \cdots, B
\end{gathered}
$$

where the matrix $\mathbf{H}[l] \in \mathbb{C}^{N \times M}$ has complex-valued normally distributed entries according to $\mathcal{C N}\left(\mu_{l}, \sigma_{l}^{2}\right)$ for every $l=0, \ldots,\left(L_{\mathrm{ch}}-1\right)$ and $\delta\left[n_{c}\right]$ is the discrete-time Dirac delta. The parameters $L_{\mathrm{ch}}, \mu_{l}$ and $\sigma_{l}^{2}$ depend on the specific channel model adopted. Then the received signal vector is given by:

$$
\mathbf{r}\left[n_{c}\right]=\sum_{l=0}^{L_{\mathrm{ch}}-1} \mathbf{H}[l] \tilde{\mathbf{s}}_{\mathrm{cp}}\left[n_{c}-l\right]+\mathbf{v}\left[n_{c}\right] \quad n_{c}=0, \ldots, N_{c}-1,
$$

where the vector $\mathbf{v}\left[n_{c}\right]=\left[v_{1}\left[n_{c}\right], v_{2}\left[n_{c}\right], \ldots, v_{N}\left[n_{c}\right]\right]^{T} \in$ $\mathbb{C}^{N \times 1}$ represents the zero-mean complex Additive White Gaussian Noise (AWGN), which has entries distributed according to $\mathcal{C N}\left(0, \sigma_{\text {noise }}^{2}\right) \forall n_{c}$. The noise variance $\sigma_{\text {noise }}^{2}$ is related to the average Signal to Noise Ratio (SNR) $\gamma_{\mathrm{AV}}$ according to $\sigma_{\text {noise }}^{2}=\gamma_{\mathrm{AV}}^{-1}$. In (10) the index $n_{c}$ identifies the $n_{c}^{\text {th }}$ sample of the TD OFDM symbol. Each component of the vector (10) may be written as:

$$
r_{n}\left[n_{c}\right]=\sum_{m=1}^{M} \sum_{l=0}^{L_{\mathrm{ch}}-1}(\mathbf{H}[l])_{n, m} \tilde{s}_{m, \mathrm{cp}}\left[n_{c}-l\right]+v_{n}\left[n_{c}\right],
$$

for every receive antenna $n=1, \ldots, N$ and every sub-carrier $n_{c}=0, \ldots,\left(N_{c}-1\right)$. In Eq. (10) the operator $(\cdot)_{n, m}$ selects the specific element of a matrix in position $(n, m)$. Upon defining the length- $N_{c}$ sequence:

$$
h_{n, m}^{\mathrm{padd}}[l]=\left\{\begin{array}{ll}
(\mathbf{H}[l])_{n, m} & l=0, \ldots, L_{\mathrm{ch}}-1 \\
0 & l=L_{\mathrm{ch}}, \ldots, N_{c}-1
\end{array},\right.
$$

we may write (11) as:

$$
r_{n}\left[n_{c}\right]=\sum_{m=1}^{M} h_{n, m}^{\mathrm{padd}}\left[n_{c}\right] \circledast N_{N_{c}} \tilde{s}_{m}\left[n_{c}\right]+v_{n}\left[n_{c}\right]
$$

for every $n=1, \ldots, N$ and every $n_{c}=0, \ldots,\left(N_{c}-1\right)$, where $\circledast N_{c}$ is the length- $N_{c}$ circular convolution operator.

The OFDM-aided STSK receiver based on these observations is shown at the bottom of Figure 1. For each receive antenna FFT-based OFDM demodulation is performed according to $R_{n}\left[n_{c}\right]=\operatorname{DFT}\left\{r_{n}\left[n_{c}\right]\right\}$, leading to:

$$
R_{n}\left[n_{c}\right]=\sum_{m=1}^{M}\left(\mathbf{H}_{\mathrm{eq}}\left[n_{c}\right]\right)_{n, m} \tilde{S}_{m}\left[n_{c}\right]+V_{n}\left[n_{c}\right],
$$

for every $n=1, \ldots, N$ and every $n_{c}=0, \ldots, N_{c}-1$. In Eq. (14) we have $V_{n}\left[n_{c}\right]=\operatorname{DFT}\left\{v_{n}\left[n_{c}\right]\right\}$ and the matrix $\mathbf{H}_{\mathrm{eq}}\left[n_{c}\right] \in \mathbb{C}^{N \times M} \forall n_{c}$ is defined as:

$$
\mathbf{H}_{\mathrm{eq}}\left[n_{c}\right]=\left[\sqrt{N_{c}} \mathrm{DFT}\left\{h_{n, m}^{\mathrm{padd}}\left[n_{c}\right]\right\}\right]_{m=1, \ldots, M n=1, \ldots, N} .
$$

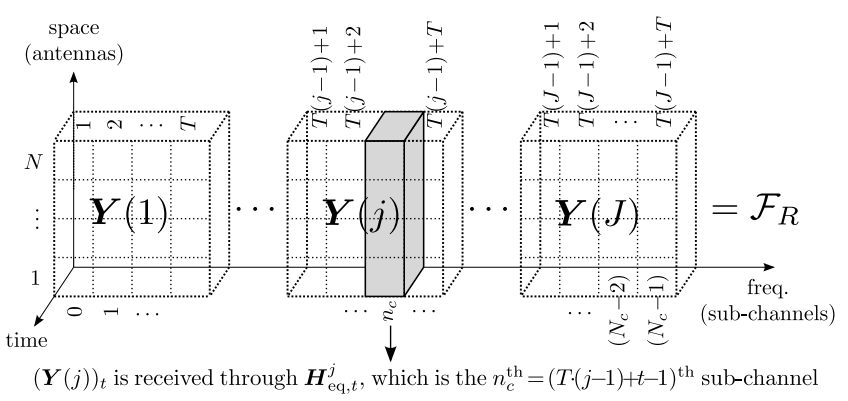

Figure 3. The $t^{\text {th }}$ column of the $j^{\text {th }}$ space-time codeword $\boldsymbol{X}(j) \in \mathbb{C}^{M \times T}$ is transmitted through the $n_{c}^{\text {th }}=(T \cdot(j-1)+t-1)^{\text {th }}$ sub-carrier, i.e. through a flat-fading sub-channel with channel gain $\boldsymbol{H}_{\mathrm{eq}, t}^{j}=\mathbf{H}_{\mathrm{eq}}[T \cdot(j-1)+t-1]$.

More particularly, $\mathbf{H}_{\mathrm{eq}}\left[n_{c}\right]$ may be viewed as the MIMO channel's frequency response, since it represents the $(N \times M)$ element MIMO channel response for each sub-carrier $n_{c}$. Eq. (14) may be rewritten in a matrix form as:

$$
\mathbf{R}\left[n_{c}\right]=\mathbf{H}_{\mathrm{eq}}\left[n_{c}\right] \tilde{\mathbf{S}}\left[n_{c}\right]+\mathbf{V}\left[n_{c}\right] \quad n_{c}=0, \ldots,\left(N_{c}-1\right),
$$

where we have $\mathbf{R}\left[n_{c}\right]=\left[R_{1}\left[n_{c}\right], R_{2}\left[n_{c}\right], \ldots, R_{N}\left[n_{c}\right]\right]^{T}$ and $\mathbf{V}\left[n_{c}\right]=\left[V_{1}\left[n_{c}\right], V_{2}\left[n_{c}\right], \ldots, V_{N}\left[n_{c}\right]\right]^{T}$. Finally, using Eq. (3), (16) may be written as:

$$
\mathbf{R}\left[n_{c}\right]=\mathbf{H}_{\mathrm{eq}}\left[n_{c}\right]\left(\boldsymbol{X}\left(\left\lceil n_{c} / T\right\rceil\right)\right)_{\left\langle n_{c}\right\rangle_{T}+1}+\mathbf{V}\left[n_{c}\right]
$$

for every $n_{c}=0, \ldots,\left(N_{c}-1\right)$. As seen from Eq. (17), each space-time codeword grouped in the frame $\mathcal{F}_{T}$ is transmitted through a set of $T$ adjacent sub-carriers ${ }^{1}$ with different channel gains $\mathbf{H}_{\mathrm{eq}}\left[n_{c}\right]$. Furthermore we may conclude that the OFDM transceiver transforms the TD spreading of the STSK in a FD spreading, since the $\mathcal{L}$-ary symbol is spread across sub-carriers and not across time slots, which is in contrast to the classic STSK scheme. More particularly, as explained in Figure 3, each column of a space-time codeword is transmitted on a sub-carrier and hence through a non-dispersive sub-channel.

Following OFDM demodulation, the space-time demapper decomposes every received frame $\mathcal{F}_{\mathrm{R}} \in \mathbb{C}^{N \times N_{c}}$ into $J$ constituent space-time codewords, leading to:

$$
\mathcal{F}_{\mathrm{R}}=\left[(\boldsymbol{Y}(1))_{1} \ldots(\boldsymbol{Y}(1))_{T} \ldots(\boldsymbol{Y}(J))_{1} \ldots(\boldsymbol{Y}(J))_{T}\right] .
$$

In Eq. (18) $\boldsymbol{Y}(j) \in \mathbb{C}^{N \times T}$ is the $j^{\text {th }}$ received space-time codeword in the frame $\mathcal{F}_{\mathrm{R}}$ and the operator $(\cdot)_{t}$ selects the $t^{\text {th }}$ column of a matrix, hence:

$$
(\boldsymbol{Y}(j))_{t}=\boldsymbol{H}_{\mathrm{eq}, t}^{j}(\boldsymbol{X}(j))_{t}+\text { noise } \quad t=1, \ldots, T .
$$

\footnotetext{
${ }^{1}$ It is also possible to carry out the mapping of bits to non-dispersive sub-carriers in different ways and it depends on both the channel-induced dispersion as well as on the Doppler frequency, what is the most efficient mapping technique. This will be studied in our future work.
} 


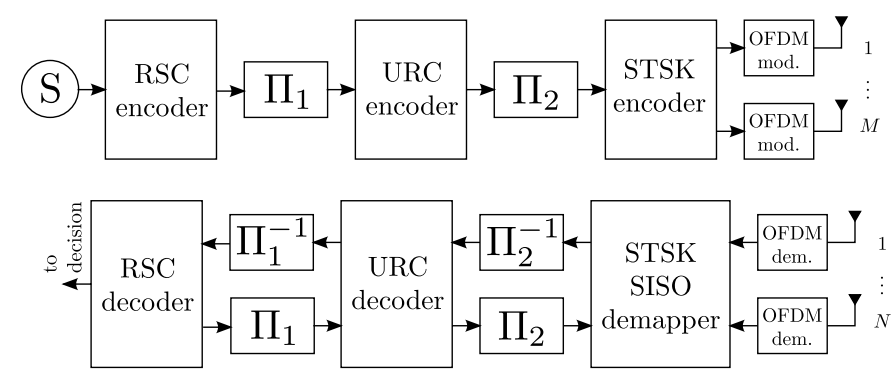

Figure 4. En/decoder for the channel-coded OFDM-aided STSK system.

where:

$$
\boldsymbol{H}_{\mathrm{eq}, t}^{j}=\mathbf{H}_{\mathrm{eq}}[T \cdot(j-1)+t-1]
$$

for every $t=1, \ldots, T$ and every $j=1, \ldots, J$.

Finally, the STSK decoder invokes the optimal singleantenna-based Maximum Likelihood (ML) decision rule similarly to the one adopted in [7] for decoding the space-time codewords with the aid of a ML detector. Specifically, the estimate $[\hat{l}(j), \hat{q}(j)]$ for the $j^{\text {th }}$ space-time codeword is given by minimizing the following metric:

$$
[\hat{l}(j), \hat{q}(j)]=\arg \min _{l, q}\left\{\left\|\overline{\boldsymbol{Y}}(j)-\overline{\boldsymbol{H}}_{\mathrm{eq}}^{j} \chi \boldsymbol{K}_{l, q}\right\|^{2}\right\},
$$

where we have:

$$
\begin{aligned}
\overline{\boldsymbol{Y}}(j) & =\operatorname{vec}(\boldsymbol{Y}(j)) \in \mathbb{C}^{N T \times 1}, \\
\boldsymbol{\chi} & =\left[\operatorname{vec}\left(\boldsymbol{A}_{1}\right) \cdots \operatorname{vec}\left(\boldsymbol{A}_{Q}\right)\right] \in \mathbb{C}^{M T \times Q}, \\
\boldsymbol{K}_{l, q} & =[\underbrace{0 \cdots 0}_{q-1}, s_{l}, \underbrace{0 \cdots 0}_{Q-q}]^{T} \in \mathbb{C}^{Q \times 1}, \\
\overline{\boldsymbol{H}}_{\mathrm{eq}}^{j} & =\left[\begin{array}{cccc}
\boldsymbol{H}_{\mathrm{eq}, 1}^{j} & \mathbf{0} & \cdots & \mathbf{0} \\
\mathbf{0} & \boldsymbol{H}_{\mathrm{eq}, 2}^{j} & \cdots & \mathbf{0} \\
\vdots & \vdots & \ddots & \vdots \\
\mathbf{0} & \mathbf{0} & \cdots & \boldsymbol{H}_{\mathrm{eq}, T}^{j}
\end{array}\right] \in \mathbb{C}^{N T \times M T}
\end{aligned}
$$

and $\mathbf{0} \in \mathbb{C}^{N \times M}$ is an $(N \times M)$-element matrix.

\section{Channel Coded Scenario}

In a serially-concatenated turbo-coded scenario a Recursive Systematic Convolutional (RSC) encoder and a UnityRate Convolutional (URC) encoder ${ }^{2}$ are introduced before the STSK encoder with the aim of reaching a near-capacity performance [7], [10]. The encoders employed have generator polynomials denoted as $\left(g, g_{f}\right)$, a constraint length of $\mathcal{K}$ and a rate of $R_{c}$. The Soft-Input Soft-Output (SISO) demapper of Figure 4 calculates the Log-Likelihood Ratios (LLRs) $L_{e}\left(x_{b}\right) b=1, \ldots, B$ of the $B$ bits of the received STSK codeword $\overline{\boldsymbol{Y}}(j)$ using the equivalent channel matrix $\overline{\boldsymbol{H}}_{\mathrm{eq}}^{j}$ and the apriori LLRs $L_{a}\left(x_{b}\right) b=1, \ldots, B$. The LLRs are calculated according to (6), where $\mathbb{K}_{x}^{b}$ represents the set of STSK codewords mapped to a block of $B$ bits, where the $b^{\text {th }}$ bit is $x$. Then the LLRs $L_{e}\left(x_{b}\right)$ are fed into the outer decoders

\footnotetext{
${ }^{2}$ The explicit benefit of using the URC encoder of Figure 4 is that it has an infinite impulse response and therefore spreads the extrinsic information efficiently without increasing the interleaver delay and hence resulting in a near capacity performance.
}

Table I

SIMULATION SYSTEM PARAMETERS

\begin{tabular}{|l|r|}
\hline$(M, N, T, Q, \mathcal{L})$ & $(2,2,2,4,4)$ \\
\hline Modulation & QPSK $(\mathcal{L}=4)$ \\
\hline$N_{c}$ & 64 or 32 \\
\hline$f_{\mathrm{d}} \tau$ & 0.001 or block fading \\
\hline $\mathcal{T}$ & $5 \cdot 10^{-8} \mathrm{~s}$ (as is in the $802.11 \mathrm{~g}$ standard) \\
\hline$R$ & $\left(g, g_{f}\right)=(2,3)_{8}, \mathcal{K}=2, R_{c}=1 / 2$ \\
\hline RSC encoder & $\left(g, g_{f}\right)=(2,3)_{8}, \mathcal{K}=2, R_{c}=1$ \\
\hline URC encoder & \\
\hline$L_{\mathrm{cp}}$ & \\
\hline$R_{\mathrm{cp}}$ & $1.48\left(N_{c}=32\right), 1.7\left(N_{c}=64\right)[$ bit $/$ symbol] \\
\hline$\left(I_{\mathrm{in}}, I_{\mathrm{out}}\right)$ & \\
\hline
\end{tabular}

according to the turbo principle, where $I_{\text {out }}$ outer iterations are performed between the RSC and the URC decoders and $I_{\text {in }}$ iterations between the URC decoder and the SISO demapper for each of the outer iterations, as further detailed in [7].

\section{E. Spreading Matrix Design}

The matrix sets employed were found by generating $N_{\text {search }}=10^{6}$ matrix sets having random elements and then selecting the best one using the capacity maximization criterion of [7]. More particularly, the search process consists of generating a set of $Q$ random matrices $\left\{\boldsymbol{A}_{q}\right\}_{q=1}^{Q}$, which have entries that are distributed according to the complex Gaussian distribution $\mathcal{C N}(0,1)$. Then each of the $Q$ matrices are normalized in order to satisfy the constraint of unity energy for each sub-carrier. Finally, the "quality" of the set generated is evaluated by calculating the corresponding Discrete-input Continuous-output Memoryless Channel (DCMC) capacity value at a fixed SNR $\overline{\gamma_{\mathrm{AV}}}$. Again, this process was repeated $N_{\text {search }}=10^{6}$ times and the set having the highest capacity was then used in our simulations.

\section{RESUlTS}

In this section the performance of the proposed system is evaluated. The parameters adopted in our Monte Carlo simulations are summarised in Table I. An OFDM-aided QPSK modulated $\operatorname{STSK}(2,2,2,4,4)$ scheme associated with a rate of $R=2$ [bit info/symbol] was adopted for characterizing our system. In the channel coded scenario a rate $R_{c}=$ $1 / 2$ RSC encoder having the octally represented generators $\left(g, g_{f}\right)=(2,3)_{8}$ and a constraint length of $\mathcal{K}=2$ as well as a URC encoder having generators $\left(g, g_{f}\right)=(2,3)_{8}$ and a constraint length of $\mathcal{K}=2$ were employed. The channel is a frequency-selective COST207 Typical Urban (TU) faded channel modelled by a FIR filter having $L_{\mathrm{ch}}=12$ taps. In the uncoded scenario the system invoked the ML detector of Equation (21), while in the coded scenario the SISO demapper of Equation (6) was adopted. We assumed to have a perfect knowledge of the MIMO channel $\mathbf{H}_{\text {eq }}\left[n_{c}\right]$ for every sub-carrier $n_{c}=0, \ldots,\left(N_{c}-1\right)$ and for every OFDM symbol. The knowledge of the frequency response $\mathbf{H}_{\mathrm{eq}}\left[n_{c}\right]$ may be acquired with the aid of a training sequence as in the $802.11 \mathrm{n}$ standard.

In the uncoded scenario, depicted in Figure 5, two different environments are considered: a frequency-selective block faded channel (i.e. a channel that remains constant during an OFDM symbol) and a frequency-selective correlated 


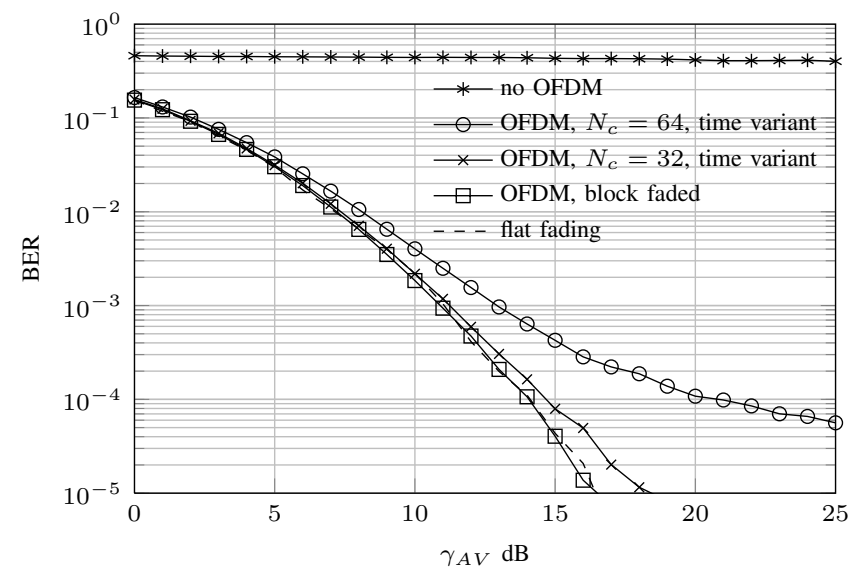

Figure 5. BER curves of the uncoded OFDM-aided QPSK modulated $\operatorname{STSK}(2,2,2,4,4)$ system for $N_{c}$ sub-carriers on a $L_{\mathrm{ch}}=12$-tap COST-207 Typical Urban time-variant $\left(N_{c}=32: \times\right.$ and $\left.N_{c}=64: \circ\right)$ and block faded ( $\square$ ) channel. The performance of a QPSK modulated $\operatorname{STSK}(2,2,2,4,4)$ system in the same frequency-selective channel $(*)$ and in a Rayleigh flat fading channel (dashed line) are also shown.

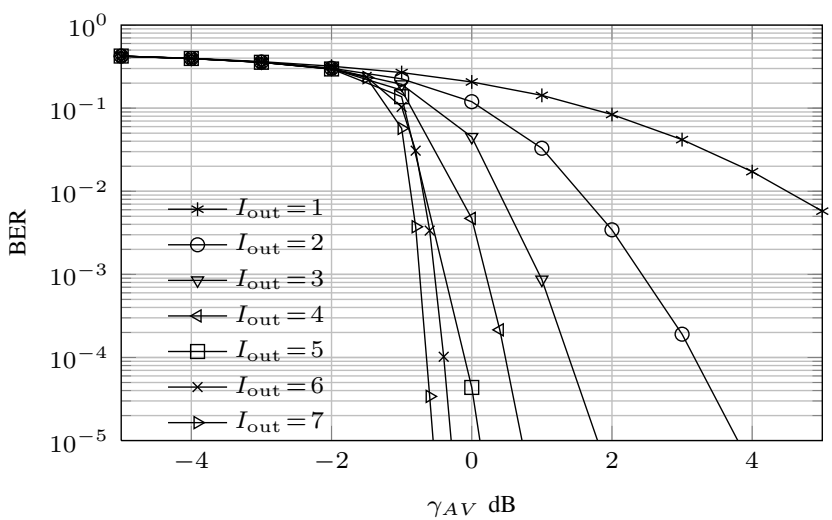

Figure 6. BER of the coded $\operatorname{OFDM}$-aided $\operatorname{STSK}(2,2,2,4,4)$ system for $N_{c}=64$ sub-carriers on a $L_{\mathrm{ch}}=12$-tap COST-207 Typical Urban block faded channel for $I_{\text {out }}=1,2, \ldots, 7$ outer iterations.

time-variant channel having a normalized Doppler spread of $f_{\mathrm{d}} \tau=0.001$. The BER curves of a QPSK modulated $\operatorname{STSK}(M, N, T, Q, \mathcal{L})=(2,2,2,4,4)$ system operating in the same frequency-selective channel and in a Rayleigh flat fading environment were also plotted as benchmarkers.

As one can see from Figure 5, the performance of the system in the correlated time-variant fading environment is influenced by the specific number of sub-carriers $N_{c}$. This may be explained by the fact that when $N_{c}$ is increased, the hypothesis of having a constant channel envelope during an OFDM symbol becomes invalid and the BER curve exhibits an error floor. In the case of a time-variant channel, the system's performance is close to that of the flat-faded STSK scenario for $N_{c}=32$ sub-carriers, albeit the effective throughput is reduced due to the $\mathrm{CP}$ overhead - see Table I. By contrast, for $N_{c}=64$ sub-carriers the BER performance exhibits an error floor, but it still remains better than that of the frequency-selective faded single-carrier STSK scenario. On the other hand, the performance of the OFDM-aided STSK scheme recorded for the idealized block-faded frequencyselective channel is virtually identical to that of the flat-faded STSK system, demonstrating the benefits of the OFDM-STSK scheme developed. Furthermore the BER performance of the system in the block-faded frequency-selective channel does not depend on the number of sub-carriers $N_{c}$, hence $N_{c}$ may be increased with the aim of reducing the relative overhead due to the adoption of the CP.

The channel coded OFDM-aided STSK scheme of Fig. 4 was investigated in the ideal case of a block-faded frequencyselective channel. As we can see from Figure 6, the proposed system is capable of attaining a vanishingly low BER even in a severely dispersive wideband channel. For $I_{\text {out }}=7$ outer iterations and $I_{\text {in }}=2$ inner iterations an infinitesimally low BER is achieved at an average SNR of $\gamma_{\mathrm{AV}}=-0.6 \mathrm{~dB}$.

\section{CONCLUSIONS AND FutURE WORK}

In conclusion, motivated by the eroded performance of the STSK scheme in a dispersive channel, a novel OFDMaided STSK system was proposed. Our results confirm that the proposed system has a similar performance to that of the single-carrier STSK scheme in a narrowband channel, when low-mobility scenarios are considered. Furthermore, the proposed scheme may attain a good performance even in correlated time-variant scenarios, provided that the system parameters are appropriately chosen. Finally, the OFDM-aided STSK system has been incorporated in a three-stage serially concatenated turbo coded scheme, leading to near capacity performance even in a broadband scenario.

The proposed OFDM-aided STSK system may be used as the basis of an OFDM-aided Space-Time-Frequency Shift Keying system, where three-dimensional space-time-frequency dispersion matrices spread a symbol of a $\mathcal{L}$-PSK/QAM constellation in the space, time and frequency domains.

\section{REFERENCES}

[1] P. W. Wolniansky, G. J. Foschini, G. D. Golden, and R. A. Valenzuela, "V-BLAST: An Architecture for Realizing Very High Data Rates over the Rich-scattering Wireless Channel," Intern. Sym. on Signals, Systems, and Electronics, 1998., pp. 295 - 300, September 1998.

[2] S. Alamouti, "A Simple Transmit Diversity Technique for Wireless Communications," IEEE Journal Selected Areas in Commun., vol. 16 , pp. 1451 - 1458, October 1998.

[3] V. Tarokh, H. Jafarkhani, and A. R. Calderbank, "Space-Time Block Codes from Orthogonal Designs," IEEE Trans. Inf. Theory, vol. 45, pp. $1456-1467$, July 1999.

[4] B. Hassibi and B. Hochwald, "High-rate Codes that are Linear in Space and Time," IEEE Trans. Inf. Theory, vol. 48, pp. 1804 - 1824, July 2002.

[5] R. Y. Mesleh, H. Haas, S. Sinanovic, C. W. Ahn, and S. Yun, "Spatial Modulation," IEEE Trans. Veh. Technol., vol. 57, pp. 2228 - 2241, July 2008.

[6] J. Jeganathan, A. Ghrayeb, L. Szczecinski, and A. Ceron, "Space Shift Keying Modulation for MIMO Channels," IEEE Trans. Wireless Commun., vol. 8, pp. 3692 - 3703, July 2009.

[7] S. Sugiura, S. Chen, and L. Hanzo, "Coherent and Differential SpaceTime Shift Keying: A Dispersion Matrix Approach," IEEE Trans. Commun., vol. 58, pp. 3219 - 3230, November 2010.

[8] H. A. Ngo, C. Xu, S. Sugiura, and L. Hanzo, "Space-Time-Frequency Shift Keying for Dispersive Channels," IEEE Signal Processing Lett., vol. 18, pp. 177-180, March 2011.

[9] L. Hanzo, M. Münster, B. J. Choi, and T. Keller, OFDM and MC-CDMA for Broadband Multi-User Communications, WLANs, and Broadcasting. John Wiley and Sons, September 2003.

[10] L. Hanzo, O. Alamri, M. El-Hajjar, and N. Wu, Near-Capacity MultiFunctional MIMO Systems: Sphere-Packing, Iterative Detection and Cooperation. Wiley-IEEE Press, May 2009. 